\title{
Light quark energy loss in a soft-wall AdS/QCD model
}

\author{
Xiangrong Zhu ${ }^{1, \text { * }}$ and Zi-qiang Zhang, \\ ${ }^{1}$ School of Science, Huzhou University, Huzhou 313000, China \\ ${ }^{2}$ School of Mathematics and Physics, China University of Geosciences, Wuhan 430074, China
}

\begin{abstract}
We investigate the energy loss of light quarks in a holographic QCD model with conformal invariance broken by a background dilaton. We perform the analysis within falling string and shooting string, respectively. It turns out that the two methods give the same result: the presence of chemical potential and confining scale tends to enhance the energy loss, in accord with previous findings of drag force and jet quenching parameter.
\end{abstract}

PACS numbers: 11.25.Tq, 11.15.Tk, 11.25-w

\section{INTRODUCTION}

One of the main purposes of the heavy-ion collisions experiments is to explore the QCD phase diagram and the properties of new state of matter produced through collisions at high energy density. It is believed that the experimental program at Relativistic Heavy-Ion Collider (RHIC) and Large Hadron Collider (LHC) have created a new state of matter so-called quark gluon plasma (QGP) [1, 2]. One of the striking features of such substance is jet quenching: the energy loss of high energy partons produced through collisions as they interact with the plasma before they fragment into hadrons (for recent reviews see [3, 4] ). On the other hand, it has been observed that QGP behaves as a strongly coupled fluid [5-7], which involves nonperturbative physics suitable for the application of the anti-de Sitter/conformal field theory (AdS/CFT) correspondence.

AdS/CFT [8 10], the duality between the type IIB superstring theory formulated on $\operatorname{AdS}_{5} \times S^{5}$ and $\mathcal{N}=4$ super Yang-Mills theory (SYM) in four dimensions, provides a useful tool for understanding the strong interaction, i.e. quantum chromodynamics (QCD). Although SYM differs from QCD in many properties at zero temperature, it reveals some qualitative features of QCD in the strongly coupled regime at non-zero temperature. During the last two decades, the AdS/CFT correspondence has yielded many important insights for studying various aspects of QGP (see [11, 12] for recent reviews with many phenomenological applications). An interesting example of such applications is jet quenching. For instance, the drag force which describes the energy loss for heavy quarks moving through $\mathcal{N}=4$ SYM plasma was studied in [13, 14]. Moreover, the jet quenching of light quarks moving through $\mathcal{N}=4 \mathrm{SYM}$ plasma has been addressed based on various approaches, e.g., jet quenching parameter [15, 16], falling string [17 21], shooting string [22, 23], etc.

Here we present an alternative holographic approach to study the energy loss of light quarks. The motivation is that AdS/QCD models, e.g., hard wall [24, 25], soft wall [26] and some improved holographic models 27 33] could provide a nice phenomenological description of quark-antiquark interaction and some hadronic properties. In particular, we will employ the $\mathrm{SW}_{T, \mu}$ model [34] which is defined by the AdS with a charged black hole to describe finite temperature and density multiplied by a warp factor to generate confinement. It turns out that this model has achieved considerable success in describing various aspects of hadron physics 34 -41]. Another motivation for this paper is that the drag force and jet quenching parameter have been studied in the $\mathrm{SW}_{T, \mu}$ model [38, 39] and the results show that the presence of chemical potential and confining scale increases the two parameters thus enhancing the energy loss. Inspired by this, we wonder whether chemical potential and confining scale have the same effect on the energy loss of light quarks using falling string and shooting string.

The outline of the paper is as follows. In the next section, we introduce the $\mathrm{SW}_{T, \mu}$ model presented in [34]. In section 3, we study the energy loss of light quarks in this model within falling string and shooting string, in turn. The last part is devoted to conclusion and discussion.

*Electronic address: xrongzhu@zjhu.edu.cn

†Electronic address: zhangzq@cug.edu.cn 


\section{SETUP}

In this section, we briefly review the $\mathrm{SW}_{T, \mu}$ model given in [34]. In the string frame, the $\mathrm{SW}_{T, \mu}$ model has the following metric

$$
d s^{2}=\frac{R^{2}}{z^{2}} h(z)\left(-f(z) d t^{2}+d \vec{x}^{2}+\frac{d z^{2}}{f(z)}\right)
$$

with

$$
f(z)=1-\left(1+Q^{2}\right)\left(\frac{z}{z_{h}}\right)^{4}+Q^{2}\left(\frac{z}{z_{h}}\right)^{6}, \quad h(z)=e^{c^{2} z^{2}}
$$

where $R$ is the AdS radius. $Q$ is the charge of black hole. $z$ represents the fifth coordinate with $z=z_{h}$ the horizon, defined by $f\left(z_{h}\right)=0$. The warp factor $h(z)$, characterizing the soft wall model, distorts the background metric and brings the confining scale $c$ (see [27] for a anatlytical way to introduce the warp factor within potential reconstruction approach).

The temperature of the black hole reads

$$
T=\frac{1}{\pi z_{h}}\left(1-\frac{Q^{2}}{2}\right), \quad 0 \leq Q \leq \sqrt{2} .
$$

The chemical potential reads

$$
\mu=\sqrt{3} Q / z_{h}
$$

Notice that for $Q=0$, the $\mathrm{SW}_{T, \mu}$ model becomes the Andreev model [42]. For $c=0$, it reduces to the AdS-Reissner Nordstrom black hole [43, 44]. For $Q=c=0$, it restores to the AdS black hole.

\section{ENERGY LOSS OF LIGHT QUARKS IN THE SW ${ }_{T, \mu}$ MODEL}

\section{A. Falling string}

In previous research, various authors [17 21] have studied the jet quenching of light quark in $\mathcal{N}=4 \mathrm{SYM}$ plasma using falling string in different ways, e.g, with or without the addition of fundamental-charge matter, specify different initial conditions. Though each approaches the subject from different vantage points, the analysis of the stopping distance traveled by the falling string in $A d S_{5}$-Schwarzschild are consistent.

Next, we will follow the argument in [20, 21] to study the jet quenching of light quark in the $\mathrm{SW}_{T, \mu}$ model by analyzing the stopping distance of an image jet induced by a massless source field, characterized by a massless particle falling along the null geodesic in the WKB approximation. According to this scenario, the R-charged current is generated by a massless gauge field in the gravity dual and the induced current is regarded as an energetic jet passing through the medium. When the wave packet of the massless gauge field falls into the horizon of the dual geometry, the image jet on the boundary dissipates and then thermalizes in the medium. The stopping distance is therefore defined as the (maximum) distance for a jet passing through the medium before it thermalizes.

In the WKB approximation, the wave packet of the massless gauge field in the gravity dual is supposed to be localized in the momentum space such that the wave function of the gauge field could be factorized as

$$
A_{j}(t, z)=\exp \left[\frac{i}{\hbar}\left(q_{k} x_{k}+\int d z q_{z}\right)\right] \tilde{A}_{j}(t, z),
$$

where $q_{k}$ denotes the 4-momentum, conserved as the metric preserves the translational symmetry along the 4 dimensional spacetime. $q_{z}$ represents the momentum along the bulk direction. $\tilde{A}_{j}(t, z)$ refers to the slow-varying with respect to $t$ and $z . j, k$ are the 4-dimensional spacetime coordinates.

In the classical limit, i.e., $\hbar \rightarrow 0$, the equation of motion of the wave pack reduces to a null geodesic,

$$
0=\left(d s^{2}\right)=d x^{i} g_{i j} d x^{j}+d z g_{z z} d z
$$

yielding

$$
\frac{d z}{d \zeta}=\frac{1}{\sqrt{g_{z z}}}\left[-g_{i j} \frac{d x^{i}}{d \zeta} \frac{d x^{j}}{d \zeta}\right]^{1 / 2}
$$



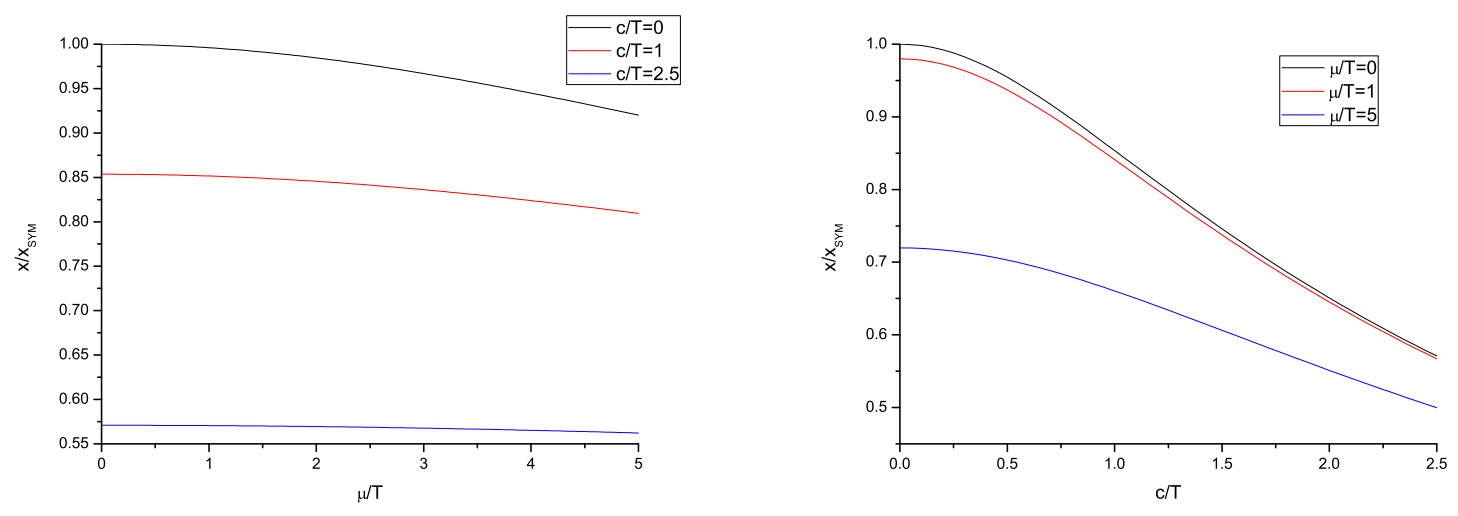

FIG. 1: Left: $x / x_{S Y M}$ versus $\mu / T$. Right: $x / x_{S Y M}$ versus $c / T$. Here we take $|\vec{q}|=0.99 \omega$.

where $\zeta$ is an affine parameter for the trajectory. As the 4-dimensional translation invariance,

$$
g_{i j} \frac{d x^{j}}{d \zeta}
$$

is conserved and proportional to $q_{i}$, leading to

$$
\frac{d x^{i}}{d \zeta} \propto g^{i j} q_{j}
$$

Then, dividing (9) by (7) gives

$$
\frac{d x^{i}}{d z}=\sqrt{g_{z z}} \frac{g^{i j} q_{j}}{\left(-q_{k} g^{k l} q_{l}\right)^{1 / 2}},
$$

one can check that the null geodesic in the above equation remains unchanged even when one uses the Einstein frame.

To proceed, we calculate the stooping distance. Supposing that the 3-momentum $\vec{q}$ to point in one of $\vec{x}$ directions, e.g., the $x_{3}$ direction, implying $q_{i}=(-\omega, 0,0,|\vec{q}|)$, where $\omega$ and $\vec{q}$ are the energy and spacial momentum of the light quark, respectively. Then plugging (10) into (10), the stopping distance for the $\mathrm{SW}_{T, \mu}$ model can be obtained,

$$
x=\int_{0}^{z_{h}} \frac{d z}{e^{c^{2} z^{2}} \sqrt{\frac{\omega^{2}}{|\vec{q}|^{2}}-\frac{1-\left(1+\frac{\mu^{2} z_{h}^{2}}{3}\right) \frac{z^{4}}{z_{h}^{4}}+\frac{\mu^{2} z^{6}}{3 z_{h}^{4}}}{e^{c^{4} z^{4}}}}},
$$

note that when one turns off the chemical potential and confining scale effects by setting $\mu=c=0$, the above equation recovers the result of SYM [20, 21].

Before going further, we need to turn to numerics. First, we determine the value range of $c$. Here we tend to study the light quark energy loss in a class of models parametrized by $c$ rather than in a specific model with fixed $c$. To that end, we make $c$ dimensionless by normalizing it at fixed $T$ and express $\mu$ in unit of it as well. The knowledge of lattice calculations suggests [45] that the range of $0 \leq c / T \leq 2.5$ is most relevant for a comparison with QCD. We take that range here.

In fig.1, we compare the stopping distance of a light quark moving in the $\mathrm{SW}_{T, \mu}$ model with the same one moving in $\mathcal{N}=4$ SYM plasma. The left panel corresponds to $x / x_{S Y M}$ versus $\mu / T$ with fixed $c / T$ while the right one represents $x / x_{S Y M}$ versus $c / T$ with fixed $\mu / T$, where $x_{S Y M}$ denotes the stopping distance of SYM. From these figures, one finds that the stopping distance in the $\mathrm{SW}_{T, \mu}$ model is smaller than that of SYM. In particular, the left panel tells us that with fixed $c / T$, increasing $\mu / T$ leads to decreasing $x / x_{S Y M}$. Namely, the inclusion of chemical potential decreases the stopping distance thus enhancing the energy loss, in accord with that found in [46]. On the other hand, one can see from the right panel that the confining scale has similar effect. Given the above, one could reach the following conclusion: the presence of chemical potential and confining scale both decrease the stopping distance thus enhancing the energy loss, consistently with the findings of the drag force [38] and jet quenching parameter [39]. 


\section{B. Shooting string}

This subsection is devoted to the analysis of the light quarks energy loss using shooting string. According to [22, 23], one considers a particular type of classical string motion: the string endpoint is close to horizon initially and move towards the boundary, carrying some energy and momentum which are gradually bled off into the rest of the string during its rise, hence, this motion is called finite-endpoint-momentum shooting string, or shooting string for short.

Next, we will follow the approach in [22, 23] to study the light quark energy loss within shooting string for the background metric (1). The instantaneous energy loss of light quarks takes the form

$$
\frac{d E}{d x}=-\frac{|L|}{2 \pi \alpha^{\prime}} \frac{1}{z^{2}}
$$

where $L$ represents the null geodesics that the endpoint follows. From the above equation, it appears that small $z$ (meaning the endpoint starts near the boundary) will result in large energy loss, indicating the jets will be quenched quickly and cannot be seen. So to hedge against this, one assumes the strings start close to the horizon.

As before, one supposes the quark moving along the $x_{3}$ direction. Given that, the energy and momentum of the quark become

$$
E=-\frac{h(z)}{\eta} \frac{f(z)}{z^{2}}
$$

and

$$
p_{x_{3}}=\frac{h(z)}{\eta z^{2}} \frac{d x}{d t}
$$

with $\eta$ the auxiliary field. Then the null geodesics reads

$$
L=\frac{E}{p_{x_{3}}}=-f(z) \frac{d t}{d x} .
$$

The finite momentum endpoints will move along $d s^{2}=0$, which gives

$$
\left(\frac{d x}{d z}\right)^{2}=\frac{1}{L^{2}-f(z)} .
$$

The denominator of (16) will vanish at $z=z_{*}$, yielding

$$
L=-\sqrt{f\left(z_{*}\right)}
$$

So the null geodesics equation becomes

$$
\frac{d x}{d z}=\frac{1}{\sqrt{f\left(z_{*}\right)-f(z)}}
$$

Finally, using (11), (12) and (17), one obtains the energy loss for the $\mathrm{SW}_{T, \mu}$ model as

$$
\frac{d E}{d x}=-\frac{e^{c^{2} z^{2}}}{2 \pi \alpha^{\prime}} \frac{\sqrt{1-\left(1+\frac{\mu^{2} z_{h}^{2}}{3}\right) \frac{z_{*}^{4}}{z_{h}^{4}}+\frac{\mu^{2} z_{*}^{6}}{3 z_{h}^{4}}}}{z^{2}},
$$

note that for $\mu=c=0$, the above equation recovers the result of SYM [22, 23].

Before going further, one needs to solve the null geodesics equation (18). However, it is difficult to solve it analytically, but it is possible numerically. The procedures of the numerical evaluation are as follows: First, sending $z_{*} \rightarrow 0$, then for a given value of $\mu / T$, one can integrate (18) and invert to get $z(x)$. Next, substituting $z(x)$ into (19) one can obtain $d E / d x$ as a function of $c / T, \mu / T$ as well as $x$.

In fig.2, we compare the instantaneous energy loss of a light quark moving in the $\mathrm{SW}_{T, \mu}$ model with its counterpart in $\mathcal{N}=4 \mathrm{SYM}$ plasma. The left panel corresponds to $(d E / d x) /(d E / d x)_{S Y M}$ versus $\mu / T$ with fixed $c / T$ while the right one denotes $(d E / d x) /(d E / d x)_{S Y M}$ versus $c / T$ with fixed $\mu / T$, where $(d E / d x)_{S Y M}$ represents the energy loss of SYM. For both panels, one finds the energy loss in the $\mathrm{SW}_{T, \mu}$ model is larger than that of SYM. In particular, increasing $\mu / T$ or $c / T$ both increase $(d E / d x) /(d E / d x)_{S Y M}$. Namely, the inclusion of chemical potential and confining scale both increase the energy loss, consistently with the analysis of the stopping distance in the last subsection. The physical significance of the results will be discussed in the next section. 

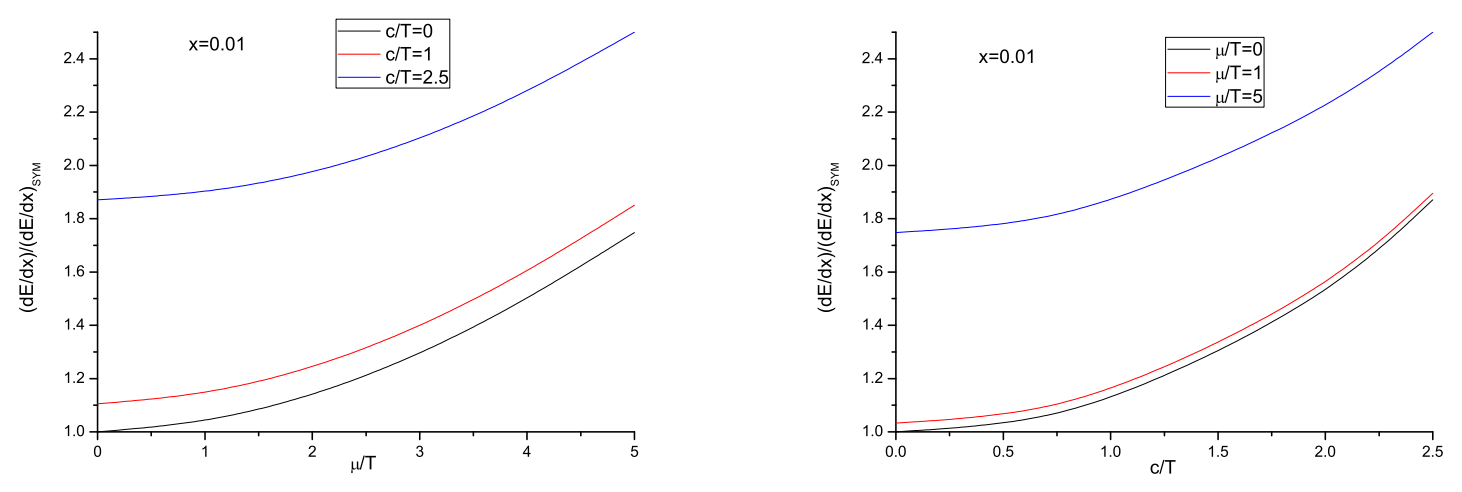

FIG. 2: Left: $(d E / d x) /(d E / d x)_{S Y M}$ versus $\mu / T$. Right: $(d E / d x) /(d E / d x)_{S Y M}$ versus $c / T$. Here we take $x=0.01$.

\section{CONCLUSION AND DISCUSSION}

Jet quenching in high-energy heavy-ion collisions can be used to probe properties of QGP. Studying the jet quenching in strongly coupled nonconformal plasma with finite quark density may shed qualitative insights into analogous questions in QCD. In this paper, we investigated the energy loss of light quarks in the $\mathrm{SW}_{T, \mu}$ model using falling string and shooting string, in turn. We discussed how chemical potential and confining scale modify the energy loss for both cases, respectively. For the former, we calculated the stopping distance of a massless particle moving along the null geodesic and found the presence of chemical potential and confining scale both decrease the stopping distance thus increasing the energy loss. For the latter, we computed the instantaneous energy loss based on the finite endpoint momentum framework and found the same result: increase chemical potential and confining scale both enhance the energy loss. These results agree with those obtained by the drag force and jet quenching parameter in the $\mathrm{SW}_{T, \mu}$ model [38, 39]. Taking all of this together, one may draw a conclusion that the effects of chemical potential and confining scale on the energy loss of heavy quarks and light quarks are consistent.

We would like to make some comment on the possible significance of our results to the current study on heavy-ion physics although such comparison may be qualitative. Recent results of the experiments at RHIC and LHC indicate that after collision, QGP expands and the jet quenching decreases with a decrease in temperature. Since chemical potential and confining scale both have the effect of increasing the energy loss, one may infer that including the two effects may lower the required temperature region in order to observe the obtained RHIC or LHC values for jet quenching.

However, it must be admitted that there are some limitations to the present study. The major disadvantage is that the $\mathrm{SW}_{T, \mu}$ model is not a bonafide solution of the classical equations of motion. Considering jet quenching in some consistent models, e.g. [27-33] would be instructive. We hope to report our progress in this regard in the near future.

\section{ACKNOWLEDGMENTS}

This work is supported by Zhejiang Provincial Natural Science Foundation of China Nos. LY19A050001, LY18A050002 and the NSFC under Grant No. 11947410.

[1] E. V. Shuryak, Nucl. Phys. A 750, 64 (2005).

[2] M. Gyulassy and L. McLerran, Nucl. Phys. A750, 30 (2005).

[3] M. Connors, C. Nattrass, R. Reed and S. Salur, Rev. Mod. Phys. 90, 025005 (2018).

[4] G. Y. Qin, X.-N. Wang, Int. J. Mod. Phys. E 24 (2015) no.11, 1530014.

[5] E. Shuryak, Prog. Part. Nucl. Phys. 53, 273 (2004).

[6] K. Adcox et al. (PHENIX Collaboration), Nucl. Phys. A757, 184 (2005).

[7] J. Adams et al. (STAR Collaboration), Nucl. Phys. A757, 102 (2005).

[8] J. M. Maldacena, Adv. Theor. Math. Phys. 2, 231 (1998).

[9] S. S. Gubser, I. R. Klebanov and A. M. Polyakov, Phys. Lett. B 428, 105 (1998). 
[10] O. Aharony, S. S. Gubser, J. Maldacena, H. Ooguri and Y. Oz, Phys. Rept. 323, 183 (2000).

[11] J. C. Solana, H. Liu, D. Mateos, K. Rajagopal, and U. A. Wiedemann, arXiv:1101.0618.

[12] O. DeWolfe, S. S. Gubser, C. Rosen and D. Teaney, Prog. Part. Nucl. Phys. 75, 86 (2014).

[13] C. P. Herzog, A. Karch, P. Kovtun, C. Kozcaz and L. G. Yafe, JHEP 07 (2006) 013.

[14] S. S. Gubser, Phys. Rev. D 74, 126005 (2006).

[15] H. Liu, K. Rajagopal and U. A. Wiedemann, Phys. Rev. Lett. 97, 182301 (2006).

[16] H. Liu, K. Rajagopal and U. A. Wiedemann, JHEP 03 (2007) 066.

[17] S. S. Gubser, D. R. Gulotta, S. S. Pufu and F. D. Rocha, JEHP 10 (2008) 052.

[18] P. M. Chesler, K. Jensen and A. Karch, Phys. Rev. D 79, 025021 (2009).

[19] P. M. Chesler, K. Jensen, A. Karch and L. G. Yaffe, Phys. Rev. D 79, 125015 (2009).

[20] P. Arnold and D. Vaman, JHEP 10 (2010) 099.

[21] P. Arnold and D. Vaman, JHEP 04 (2011) 027.

[22] A. Ficnar and S. S. Gubser, Phys. Rev. D 89, 026002 (2014).

[23] A. Ficnar, S. S. Gubser and M. Gyulassy, Phys. Lett. B 738, 464 (2014).

[24] J. Erlich, E. Katz, D. T. Son and M. A. Stephanov, Phys. Rev. Lett. 95, 261602 (2005).

[25] J. Polchinski, M. J. Strassler, JHEP 05 (2003) 012.

[26] A. Karch, E. Katz, D. T. Son and M. A. Stephanov, Phys. Rev. D 74, 015005 (2006).

[27] J. P. Shock, F. Wu, Y-L. Wu and Z-F. Xie, JHEP 03 (2007) 064.

[28] A. Stoffers and I. Zahed, Phys. Rev. D 83, (2011) 055016.

[29] D. n. Li and M. Huang, JHEP 11 (2013) 088.

[30] D. n. Li, S. He, M. Huang and Q. S. Yan, JHEP 09 (2011) 041.

[31] S. He, M. Huang and Q. S. Yan, Phys. Rev. D 83, 045034 (2011).

[32] S. He, S. Y. Wu, Y. Yang and P. H. Yuan, JHEP 04 (2013) 093.

[33] R. Rougemont, A. Ficnar, S. Finazzo and J. Noronha, JHEP 04 (2016) 102.

[34] P. Colangelo, F. Giannuzzi and S. Nicotri, Phys. Rev. D, 83, 035015 (2011).

[35] C. Park, D.-Y. Gwak, B.-H. Lee, Y. Ko and S. Shin, Phys. Rev. D 84, (2011) 046007.

[36] P. Colangelo, F. Giannuzzi and S. Nicotri, JHEP 05 (2012) 076.

[37] P. Colangelo, F. Giannuzzi, S. Nicotri and F. Zuo, Phys. Rev. D 88 (2013) 115011.

[38] Y. Xiong, X. Tang, and Z. Luo, Chin. Phys. C 43, 113103 (2019).

[39] X. R. Zhu and Z.-q. Zhang, Chin. Phys. C 44, 105105 (2020).

[40] X. Chen, S.-Q. Feng, Y.-F. Shi, Y. Zhong, Phys. Rev. D 97, 066015 (2018).

[41] Z.-q. Zhang and X. R. Zhu, Phys. Lett. B 793 (2019) 200.

[42] O. Andreev and V. I. Zakharov, Phys. Rev. D 74, 025023 (2006).

[43] M. Cvetic, et al., Nucl. Phys. B 558 (1999) 96.

[44] D. T. Son and A. O. Starinets, JHEP 03 (2006) 052.

[45] H. Liu, K. Rajagopal and Y. Shi, JHEP 08 (2008) 048.

[46] E. Caceres, A. Kundu and D. L. Yang, JHEP 03 (2014) 073 